\title{
Application in Feature Extraction of AE Signal for Rolling Bearing in EEMD and Cloud Similarity Measurement
}

\author{
Long Han, ${ }^{1,2}$ Chengwei Li, ${ }^{1}$ and Liqun Shen ${ }^{1}$ \\ ${ }^{1}$ School of Electrical Engineering and Automation, Harbin Institute of Technology, Harbin 150001, China \\ ${ }^{2}$ School of Electrical and Control Engineering, Heilongjiang University of Science and Technology, Harbin 150022, China \\ Correspondence should be addressed to Long Han; yazhoulong@hit.edu.cn
}

Received 20 May 2015; Revised 27 July 2015; Accepted 10 August 2015

Academic Editor: José A. Becerra Villanueva

Copyright (c) 2015 Long Han et al. This is an open access article distributed under the Creative Commons Attribution License, which permits unrestricted use, distribution, and reproduction in any medium, provided the original work is properly cited.

\begin{abstract}
Due to the powerful ability of EEMD algorithm in noising, it is usually applied to feature extraction of fault signal of rolling bearing. But the selective correctness of sensitive IMF after decomposition can directly influence the correctness of feature extraction of fault signal. In order to solve the problem, the paper firstly proposes a new method on selecting sensitive IMF based on Cloud Similarity Measurement. By comparing this method in simulation experiment with the traditional mutual information method, it is obvious that the proposed method has overcome the misjudgment in the traditional method and it has higher accuracy, by factually collecting the normal, damage, and fracture fault AE signal of the inner ring of rolling bearing as samples, which will be decomposed by EEMD algorithm in the experiments. It uses Cloud Similarity Measurement to select sensitive IMF which can reflect the fault features. Finally, it sets the Multivariate Multiscale Entropy (MME) of sensitive IMF as the eigenvalue of original signal; then it is classified by the SVM to determine the fault types exactly. The results of the experiments show that the selected sensitive IMF based on Cloud Similarity Measurement is effective; it can help to improve the accuracy of the fault diagnosis and feature extraction.
\end{abstract}

\section{Introduction}

Rolling bearing is the most common part of the rolling mechanism, and about $30 \%$ of the mechanical faults are caused by rolling bearing, so the detection and diagnosis of rolling bearing are always hot issues studied by scholars all over the world. In the recent ten years, the technology of acoustic emission (AE) is important in monitoring the states of the rolling bearing; it can help to effectively test the early symptom of pitting corrosion defect and the crack initiation so as to avoid occurring disastrous consequences $[1,2]$. Elforjani has completed a lot of researches and experiments on natural defects of bearing; the results show that $\mathrm{AE}$ technology can provide us with successful detecting and monitoring of natural crack initiation and propagation in slow-speed rotating bearing $[3,4]$. Al-Ghamdi et al. have compared the RMS value, amplitude value, and kurtosis value of $\mathrm{AE}$ signal and vibration signal for the outer race fault of rolling bearing; they think that the technology of acoustic emission is more effective than the vibration in early fault diagnosis [5]. Researchers from China and abroad have discussed and explored a lot on the feature extraction methods of acoustic emission signals of bearings. Choudhury and Tandon have applied two ways of parametric analysis based on ring down counts and peaks to analyze the failures of rollers and the inner ring of bearings under the conditions of load change, fault size, and speed change [6]. Beck et al. have studied physical properties in material fracturing; the test results indicate that the relationship between $\mathrm{AE}$ energy and the fracture area or depth is linear dependence [7]. In detecting bearing experiments, Kilundu et al. have written the record of cycle stationary $\mathrm{AE}$; the results indicate that comparing the cyclic spectrum related index to the traditional time index it is more sensitive in continuously monitoring the fracture [8].

Due to bad working conditions of mechanical equipment, the collected AE signal from the spot is often polluted by all kinds of noises. To eliminate noise of the signal, some researchers have introduced the wavelet noise reduction technology into feature extraction of AE signal, and it has 
achieved good effects. Zhao et al. have decomposed the $\mathrm{AE}$ into different frequency bands by using wavelet analysis technique and reconstructed the signal, eliminated noise, and well separated faint $\mathrm{AE}$ signal [9]. Lilin et al. have extracted eigenvalue of $\mathrm{AE}$ signal by using wavelet packet energy spectrum; it can help to improve the SNR of the signal [10]. However, this method has the difficulties in selecting the wavelet base and determining the threshold. The Empirical Mode Decomposition (EMD) does not have fixed basis formula, which could avoid the difficulties in selecting wavelet base in wavelet analysis; it is more effective in nonstationary signal denoising than any other methods of wavelet transform [11]. But EMD method has modal mixing problem; it can cause the distortion of the decomposed Intrinsic Mode Function (IMF). In order to solve the frequency mixing problem in EMD denoising, Wu and Huang have proposed Ensemble Empirical Mode Decomposition (EEMD) to perform signal decomposition to enhance the thoroughness of denoising [12]. Only a part of the IMF after decomposing by EEMD is closely related to the original fault information. So it is significant for the proper selecting of the sensitive IMF which is closely related to the fault information in improving the accuracy of feature extraction of fault signal. In literatures [13-15], the sensitivity evaluation algorithm, cross-correlation coefficient, and mutual information (MI) algorithms are used to select the sensitive IMFs from all IMFs, and the sensitive IMFs could reflect the fault features. It does help to remove the illusive component, but the misjudgment still existed.

In view of the above, this paper proposes a solution of Cloud Similarity Measurement (CSM). CSM from cloud mode is used to describe the differences between clouds [16]. In data mining, CSM in similarity measurement of two time series could overcome the disadvantages in Euclidean distance, Dynamic Time Warping (DTW) distance, and pattern distance of classical methods, so as to achieve better metrics accuracy [17]. Meanwhile, CSM is extensively used in the e-business trade, biomedicine, and watermarking technology. But it has never be reported in the field of feature extraction of AE signal of rolling bearing. In view of this, in this paper, CSM is adopted to select the sensitive IMFs which could reflect the fault features from IMFs after being decomposed by EEMD for the first time. It can help to overcome the misjudgment of traditional method and improve the accuracy in selecting sensitive IMFs, which are calculated by Multivariate Multiscale Entropy (MME), so as to obtain the eigenvalues. All the above can effectively extract the fault characteristic information of AE signal and improve the accuracy of fault diagnosis.

\section{AE Signal Feature Extraction Theory of Rolling Bearing}

2.1. EEMD Algorithm. EEMD algorithm is an auxiliary signal processing method in dealing with noises. The nature of the algorithm, a Gaussian white noise, is imposed on the signals, and the mixed signals are calculated repeatedly by Empirical Mode Decomposition. After adding noise, the signal continuity may be achieved in different frequencies regions due to the evenly distributed statistical properties of Gaussian white noise frequencies, in order to reduce the IMF component model mixing degree. EEMD algorithm is showed as follows [18].

(1) To set the overall average number $M$ and standard deviation of white noise $k$.

(2) To do $m$ times EMD experiments after adding white noise. Consider the following:

(i) After a random Gaussian white noise $n_{m}(t)$ is added in the input signal $x(t)$, and the signal $x_{m}(t)$ is obtained,

$$
x_{m}(t)=x(t)+k n_{m}(t) .
$$

(ii) $x_{m}(t)$ is decomposed by EMD, to obtain $c_{j, m} \cdot c_{j, m}$ which stands for the $j$ IMF is obtained in the $m$ th decomposition $\left(j=1,2, \ldots, N_{m}\right)$, where $N_{m}$ stands for the number of IMFs in the $m$ th decomposition.

(iii) If $m<M$, let $m=m+1$; then return to (ii).

(iv) Take the minimum of model components in each group of IMFs which is obtained in the $M$ times of decompositions as the final overall average number of IMFs.

(3) Each IMF in $m$ times of decomposition being averaged:

$$
\bar{c}_{j}=\frac{\sum_{m=1}^{M} c_{j, m}}{M} \quad\left(j=1,2, \ldots, N_{m}, m=1,2, \ldots, M\right) .
$$

(4) To output $\bar{c}_{j}$ as the $j$ th IMF obtained after EEMD decomposition. The added white noise $n_{m}(t)$ is generated randomly in each experiment. When $M$ is bigger, the overall average of the added Gaussian white noise is closer to zero.

2.2. Cloud Similarity Measurement. Cloud Similarity Measurement (CSM) consists of backward cloud generator algorithm and includes angle cosine of cloud eigenvector. The input sample point $x_{i}=\left(x_{1}, x_{2}, \ldots, x_{N}\right)$ and sample point $y_{j}=\left(y_{1}, y_{2}, \ldots, y_{M}\right)$, where $N$ and $M$ are the numbers of $x_{i}$ and $y_{j}$, respectively. The steps are as follows [19].

(1) Sample mean $\bar{X}=(1 / n) \sum_{i=1}^{n} x_{i}$ is obtained based on the sample point $x_{i}$. The first order of sample absolute center distance is $(1 / n) \sum_{i=1}^{n}\left|x_{i}-\bar{X}\right|$; sample variance is $S^{2}=(1 /(n-$ 1)) $\sum_{i=1}^{n}\left(x_{i}-\bar{X}\right)^{2}$.

(2) To calculate expected value $E_{x}=\bar{X}$.

(3) To calculate entropy $E_{n}=\sqrt{\pi / 2} \times(1 / n) \sum_{i=1}^{n}\left|x_{i}-E_{x}\right|$.

(4) To calculate hyperentropy $H_{e}=\sqrt{S^{2}-E_{n}^{2}}$.

(5) To calculate cloud vector $\vec{v}_{i}=\left(E x_{i}, E n_{i}, H e_{i}\right)$ of sample point $x_{i}$ and cloud vector $\vec{v}_{j}=\left(E x_{j}, E n_{j}, H e_{j}\right)$ of sample point $y_{j}$.

(6) The similarity of any two samples $x_{i}$ and $y_{j}$ may be expressed by the included angle cosine between $\vec{v}_{i}$ and $\vec{v}_{j}$, as follows:

$$
\operatorname{sim}(i, j)=\cos \left(\vec{v}_{i}, \vec{v}_{j}\right)=\frac{\vec{v}_{i} \times \vec{v}_{j}}{\left\|\vec{v}_{i}\right\|\left\|\vec{v}_{j}\right\|} .
$$




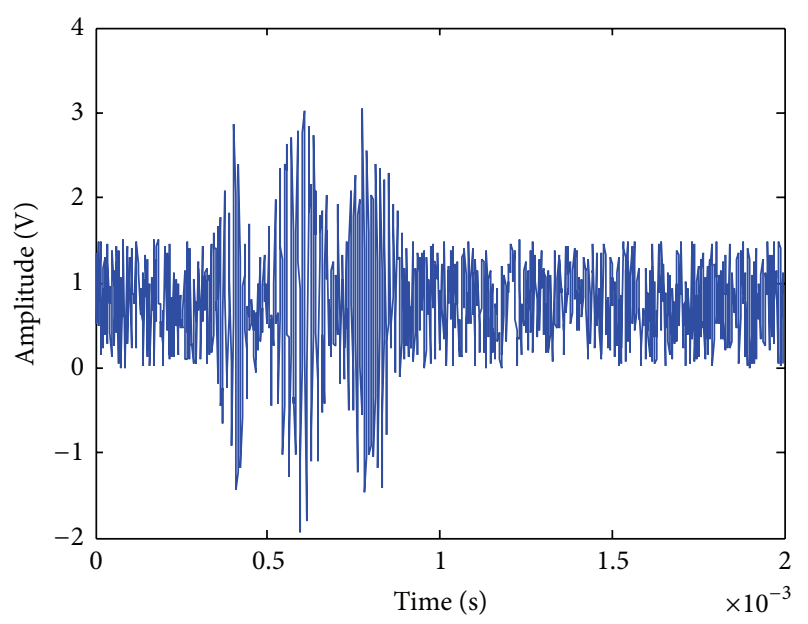

Figure 1: Analog AE signal.

To set similarity threshold to be $\delta=0.95$, to retain $\mathrm{IMF}_{j}$ when $\cos \left(\vec{v}, \vec{v}_{j}\right) \geq 0.95$, the others are removed.

2.3. The Comparison of EEMD-CSM Algorithm. Mitraković et al. [20] and other scholars use damped exponential signals with three different frequency and attenuation coefficients to simulate AE signal; the signal model is given as follows:

$$
f(x)=\sum_{i=1}^{3} A_{i} e^{\left[-a_{i}\left(t-t_{i}\right)^{2}\right]} \sin \left[2 \pi f_{i}\left(t-t_{i}\right)\right] .
$$

In this equation, $A_{i}, a_{i}, t_{i}$, and $f_{i}$ are the $i$ th harmonic signal amplitude, attenuation coefficient, peak instant, and the characteristic frequency, respectively. The parameters of typical AE signal are valued as follows: $A_{i}=2(i=1,2,3)$, $a_{1}=6.24 \times 10^{8}, a_{2}=1.56 \times 10^{8}, a_{3}=2.79 \times 10^{8}, t_{1}=0.4 \mathrm{~ms}$, $t_{2}=0.6 \mathrm{~ms}, t_{3}=0.8 \mathrm{~ms}, f_{1}=70 \mathrm{KHz}, f_{2}=60 \mathrm{KHz}$, and $f_{3}=$ $80 \mathrm{KHz}$. In fact, the noise signals are mainly white noise. Therefore, in the case of the sampling frequency $f=500 \mathrm{kHz}$, white noise signal of limited bandwidth is added in the analog AE signal, as is shown in Figure 1. The EEMD-CSM algorithm is proposed by this paper for simulating the analog AE signal to validate the effectiveness and accuracy of sensitive IMF selected by the CSM.

In EEMD algorithm, the value of added noise is 0.01 times bigger than the standard deviation, and the overall average time is 200. In the simulated AE signal after the decomposition of EEMD, IMF1 IMF4 are obtained, as is shown in Figure 2.

As can be seen in Figure 2, the IMF1 and IMF2 of EEMD decomposition are actual original signal component in which the noise interference is eliminated. IMF3 and IMF4 are meaningless illusive components, which will be excluded in subsequent analysis. The comparison between the mutual information and cloud similarity of each IMF and original signal is shown in Table 1. According to the literature [21], the calculated mutual information (MI) threshold is 0.0357; then IMF1, IMF2, and IMF3 are considered to be actual components; IMF4 component is illusive. Therefore, it could
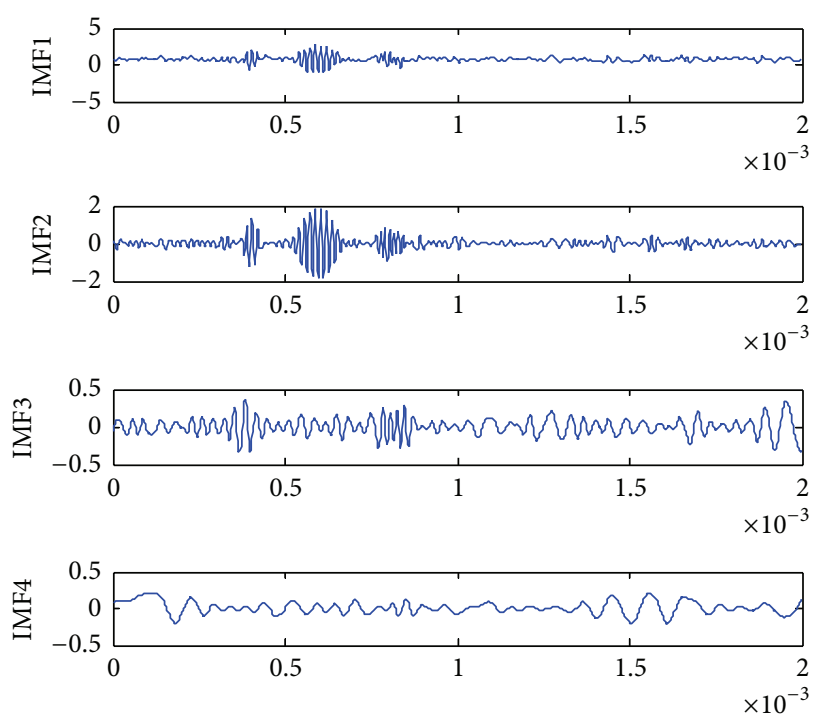

FIGURE 2: IMF1 IMF4 obtained in EEMD decomposition.

TABLE 1: Comparison between the mutual information and cloud similarity of each IMF and original signal.

\begin{tabular}{lcccc}
\hline & IMF1 & IMF2 & IMF3 & IMF4 \\
\hline Mutual information & 0.1175 & 0.0998 & 0.0363 & 0.028 \\
Cloud similarity & 0.9653 & 0.9551 & 0 & 0 \\
\hline
\end{tabular}

easily cause misjudgment when the mutual information excludes illusive component. In view of threshold $\delta=0.95$ of CSM, it is apparent that IMF3 and IMF4 are illusive, while the other two actual components are retained. Therefore, it is effective to select sensitive IMF by the CSM, which is higher than the accuracy of the mutual information method and overcomes the misjudgment.

2.4. Feature Extraction Method Based on EEMD-CSM-MME. Above all, feature extraction method based on EEMD-CSMMME can be summarized as the following steps.

(1) To obtain calculated vectors $\vec{v}=(E x, E n, H e)$ of signal $v$ by backward cloud generator algorithm.

(2) To obtain $\operatorname{IMF}_{j}(j=1,2, \ldots, n)$, after decomposing of the signal $v$ by EEMD.

(3) To obtain calculated vectors $\vec{v}_{j}=\left(E x_{j}, E n_{j}, H e_{j}\right)$ of signal IMF $\mathrm{IMy}_{j}$ using backward cloud generator algorithm.

(4) To obtain calculated $\cos \left(\vec{v}, \vec{v}_{j}\right)=\vec{v} \times \vec{v}_{j} /\|\vec{v}\|\left\|\vec{v}_{j}\right\|$, to determine the similarity of cloud vector of signal $v$ and $\mathrm{IMF}_{j}$, to set similarity threshold to be $\delta=0.95$, to retain $\mathrm{IMF}_{j}$ as the sensitive IMF, when $\cos \left(\vec{v}, \vec{v}_{j}\right) \geq 0.95$.

(5) To calculate the entropy of selected sensitive $\mathrm{IMF}_{j}$ by MME algorithm and set the obtained value of multivariate samples as eigenvalue. The formula for calculation of MME is shown as follows [22]:

$$
\operatorname{MSampEn}(M, \lambda, r, N)=-\ln \left[\frac{B^{m+1}(r)}{B^{m}(r)}\right] .
$$

In the formula, to embed vector $M=\left[m_{1}, m_{2}, \ldots, m_{p}\right]$, time lag vector $\lambda=\left[\lambda_{1}, \lambda_{2}, \ldots, \lambda_{p}\right]$, tolerance level $r$, and 


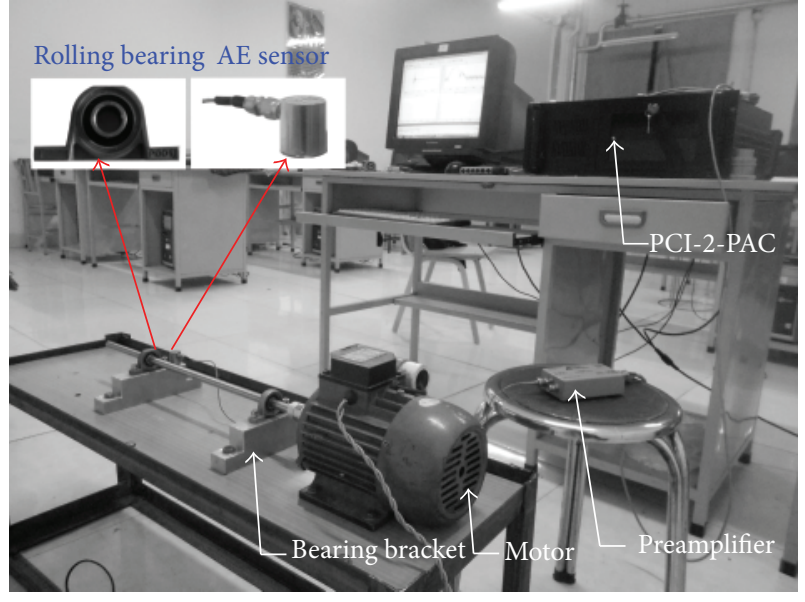

FIGURE 3: Rolling bearing experimental platform.

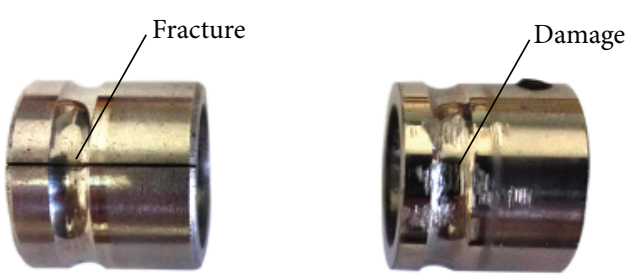

FIGURE 4: Fault types of inner ring of bearing.

length of time series $N, B^{m}(r)$ indicates the similar possibility of any two complex time lag vectors in $m$ dimensions, $B^{m+1}(r)$ indicates the similar possibility of the two vectors in $m+1$ of dimensions in the above formula.

\section{Rolling Bearing Fault Analysis}

3.1. Test-Rig Design and Layout. In order to complete simulation experiment of three conditions of the normal, damage, and fracture state of inner ring of bearing, the rolling bearing experimental test platform is shown in Figure 3. It consists of motor, coupling, testing bearing, bearing, and support structure. The type of rolling bearing produced by Chinese Nanjing haning Bearing Manufacturing Co., Ltd., is K001. The depth of rift and injury of bearing sample is $0.25 \mathrm{~mm}$, which is processed by electrical discharge. Physical samples are shown in Figure 4.

3.2. Instrumentation. The test instrument used in the experiment is using four-channel signal acquisition system of PCI2-PAC produced by American Acoustic Physics Company; the acoustic sensor is $\mathrm{R} 15 \alpha$, whose response frequency is $60 \sim$ $500 \mathrm{kHz}$ and service temperature is $-20 \sim 80^{\circ} \mathrm{C}$. The acoustic sensor is fixed to the stents, close to the bearing by M20 magnetic fixture. Then to connect the acoustic sensor to the data acquisition system with preamplifier $(40 \mathrm{~dB})$, and the output impedance of preamplifier $50 \Omega$, the working frequency is $10 \mathrm{KHz} 2 \mathrm{MHz}$. The data acquisition systems use AEwin3.5 software in data acquisition and analysis. The
TABLE 2: Cloud similarity values between original signal and IMF1 IMF7.

\begin{tabular}{lccccccc}
\hline & IMF1 & IMF2 & IMF3 & IMF4 & IMF5 & IMF6 & IMF7 \\
\hline $\begin{array}{l}\text { Cloud } \\
\text { similarity } \\
\text { values }\end{array}$ & 0.9981 & 0.9876 & 0.9556 & 0.8904 & 0 & 0 & 0 \\
\hline
\end{tabular}

speed of motor rotating is $14000 \mathrm{r} / \mathrm{min}$ and the sampling rate is 1MSPS during the experiments. Schematic diagram of data acquisition process is shown in Figure 5.

3.3. Feature Extraction of AE Signal. To respectively test the three states of acoustic emission signal of bearing's inner ring from the fault experimental platform, including the normal, fracture, and damage states, the time domain waveform is, respectively, shown in Figures 6-8.

This paper studies damage fault of bearing's inner ring; for example, it illustrates the process of feature extraction of $\mathrm{AE}$ signal. Firstly it decomposes the AE signal of inner damage fault by EEMD algorithm. In EEMD algorithm, the value of added noise is 0.01 times more than the standard deviation, and the overall average time is 200 . The result decomposed by EEMD algorithm is shown in Figure 9. To calculate the cloud similarity values between the original signal and IMF1 IMF7 by using CSM, the similarity values are shown in Table 2 .

The threshold of cloud similarity is set to be 0.95 by the present method according to the algorithm proposed by the paper, as can be seen in Table 2; the cloud similarity values between original signal and IMF1 IMF3 are bigger than 0.95; it indicates that IMF1 IMF3 are closely related to the original signal. The cloud similarity values between the remaining components and original signal are smaller than 0.95 ; it indicates that they are not so closely related to the original signal, and it will be judged to be illusive and removed. In order to further observe the distribution laws of cloud similarity values and universality of the threshold selection, to set 20 group damage samples and let them be calculated by EEMD, and to obtain the cloud similarity values between original signal and IMF1 IMF7, the fitting curves of the cloud similarity values of all damage samples are shown in Figure 10.

The marks of $\mathrm{A} \sim \mathrm{D}$, respectively, represent the fitting curves of the cloud similarity values between original signal and IMF1 IMF4 of each sample. E represents the fitting curve of the cloud similarity values between original signal and IMF5 IMF7 of each sample in Figure 10. As can be seen in Figure 10, the cloud similarity values of the fitting curves A, B, and $\mathrm{C}$ have some fluctuation, but all the values are bigger than 0.95; this shows that the original signal and IMF1, IMF2, and IMF3 are sensitive IMFs, and they can completely reflect the characteristic information of the original signal. The cloud similarity values of the fitting curves $\mathrm{D}$ and $\mathrm{E}$ are smaller than 0.95, which shows that IMF4 IMF7 have no relation with the original signal; they need to be removed.

To take 20 group samples, respectively, in normal and fracture failure state, so as to calculate the cloud similarity values of each separately, the obtained fitting curves are 


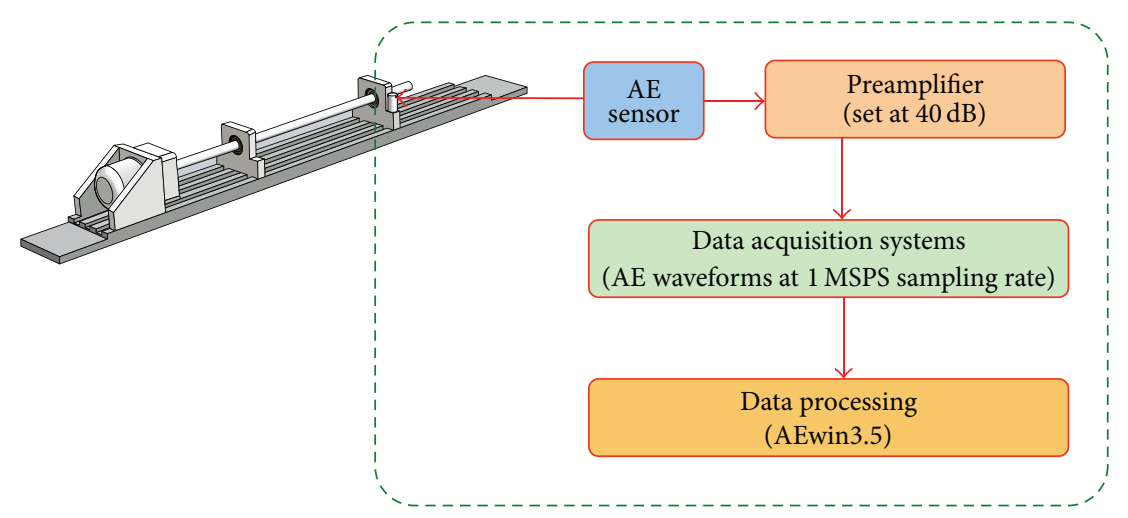

FIGURE 5: Schematic of the data acquisition systems.

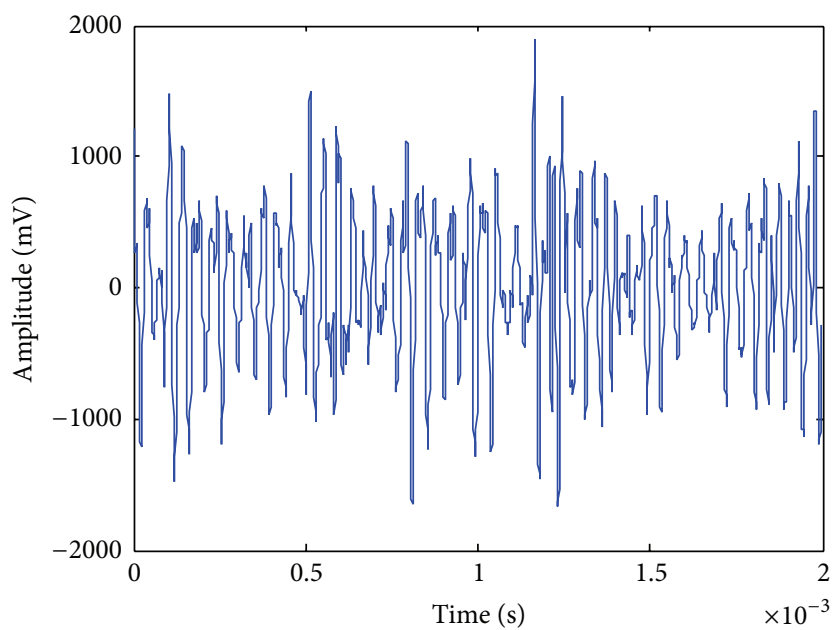

FIGURE 6: Normal signal waveform.

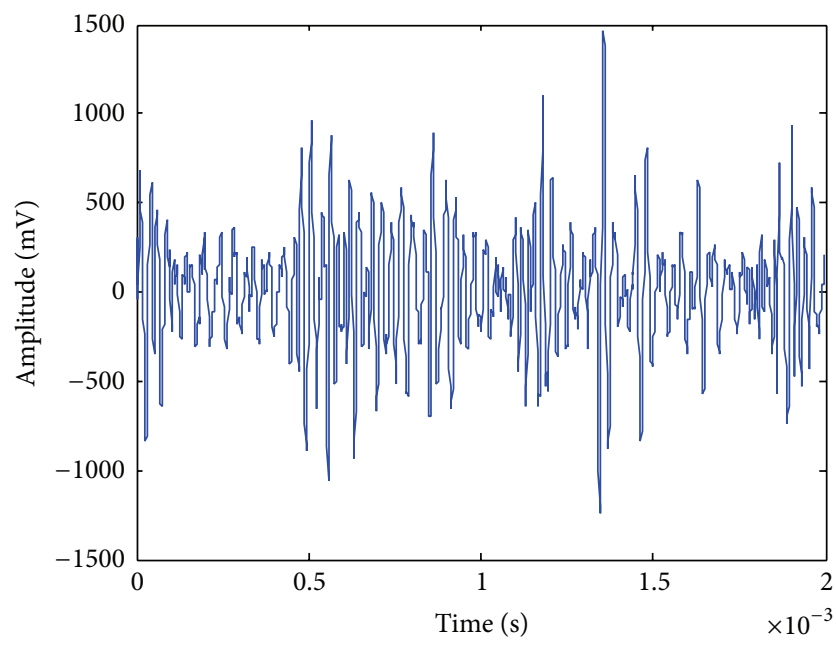

FIGURE 7: Damage signal waveform.

shown in Figures 11 and 12. As shown in Figures 11 and 12, the cloud similarity values under the two situations share the same distribution laws with the cloud similarity values of the damage samples, which means the cloud similarity values between original signal and IMF1, IMF2, and IMF3 are bigger

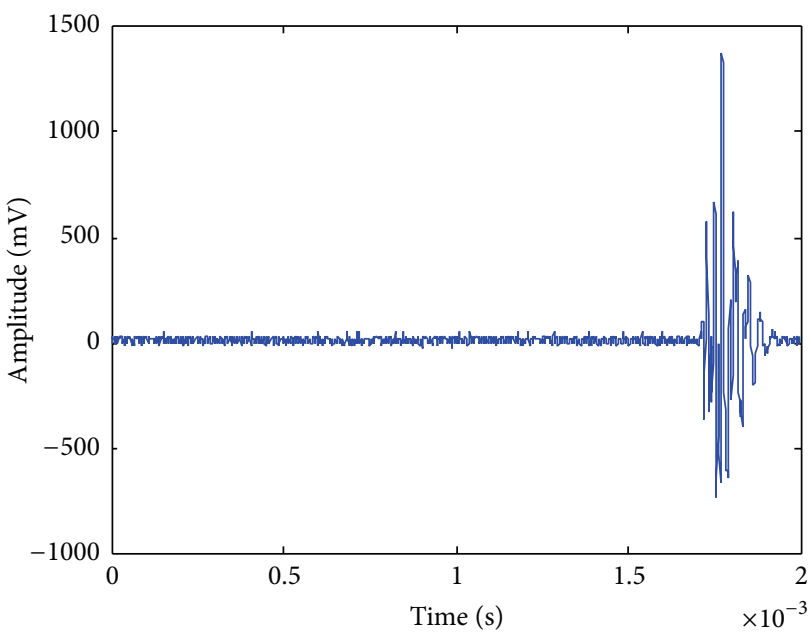

FIGURE 8: Fracture signal waveform.

than 0.95 ; the others are smaller than 0.95 . So it is suitable to select 0.95 as the threshold.

For the multiple variables composed of sensitive IMFs, MME algorithm should be used in calculating eigenvalue, where $m_{k}=2$ and $\lambda_{k}=1$; that is; $M=\left[\begin{array}{lll}2 & 2 & 2\end{array}\right], \lambda=$ $\left[\begin{array}{lll}1 & 1 & 1\end{array}\right]$, and $r=0.15 \times$ (normalized standard deviation of time series). To take three groups of AE signal samples under three states separately in the bearing inner ring, the obtained curve changes of MME are shown in Figure 13. As shown in Figure 13, the multiple sample entropy of fracture signal at 20 scales is always the minimal one, which means when fracture fault occurs, the complexity of AE signal is the lowest. The values of multiple sample entropy of normal state and damage state at 20 scales are relatively bigger; it shows the higher complexity of AE signal. There are obvious differences among the three values of multiple sample entropy, and it can be distinguished easily.

3.4. Comparison of the Fault Diagnosis Results. To set the most optimal multiple sample entropy of scale factors 9 and 15 are obtained by calculation with one-factor analysis of variance. So as the eigenvalue, it will be classified by the support vector machine (SVM) algorithm. The sample 


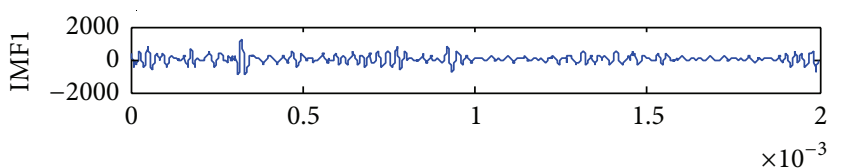

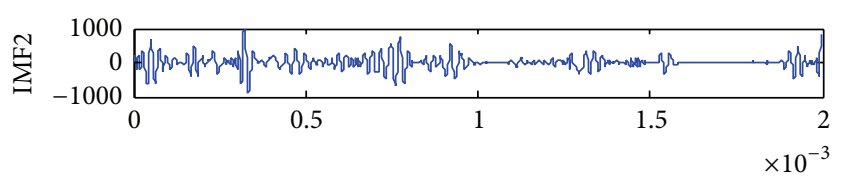

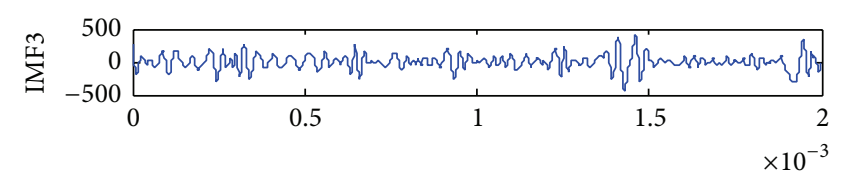$$
\sum_{-500}^{0} \begin{aligned}
& 0.5 \\
& 0
\end{aligned}
$$
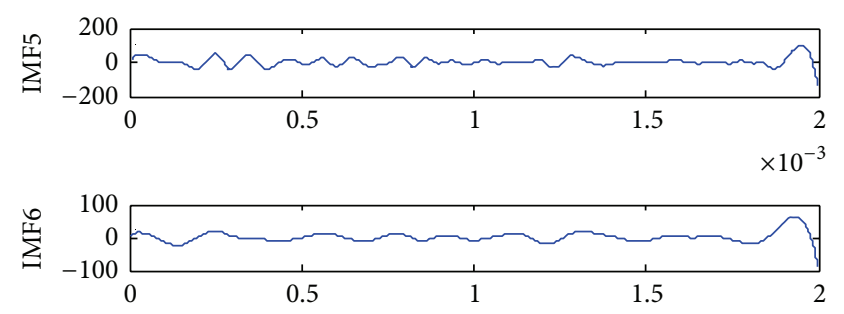

$\times 10^{-3}$

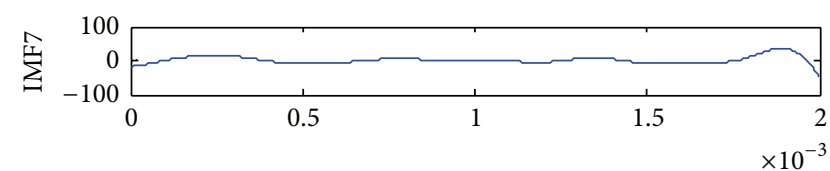

Figure 9: Damage signal decomposition results.

number of eigenvalues of the signal under normal, fracture failure, and damage state is a total of 180, among which 120 are for training samples and 60 are for testing samples. The classification results can be divided into three categories. "1" represents normal state, "0" represents damage state, and " 1 " represents the fracture failure state. When the penalty factor $C$ is $150, \sigma$ is 1 ; the fault diagnosis is shown in Figure 14(a). MI algorithm is used to select the sensitive IMFs from all IMFs, and the sensitive IMFs are calculated by the MME; then the eigenvalues are obtained, and they can be classified by SVM algorithm; the results of fault diagnosis are shown in Figure 14(b).

As shown in Figure 14, the accuracy of EEMD-CSMMME-SVM algorithm is higher. In order to further illustrate the effect of the EEMD-CSM-MME-SVM algorithm, a certain amount of testing samples is added into the original experiments. Meanwhile, the EEMD-CSM-MME-SVM and EEMD-MI-MME-SVM are used to classify the faults. The fault diagnosis results are listed in Table 3.

As shown in Table 3, when the effect of fault diagnosis by EEMD-CSM-MME-SVM algorithm is good, the accuracy rate can be $99 \%$, while when the effect of fault diagnosis by EEMD-MI-MME-SVM algorithm is not so good, the accuracy rate is only $78 \%$. After comparison, it can be

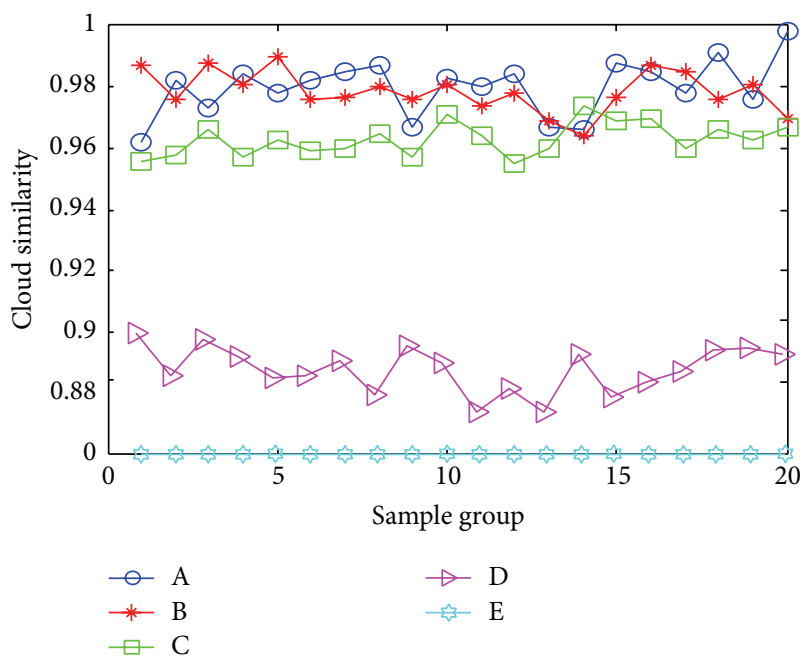

FIGURE 10: The fitting curves of cloud similarity values of 20 groups of damage samples.

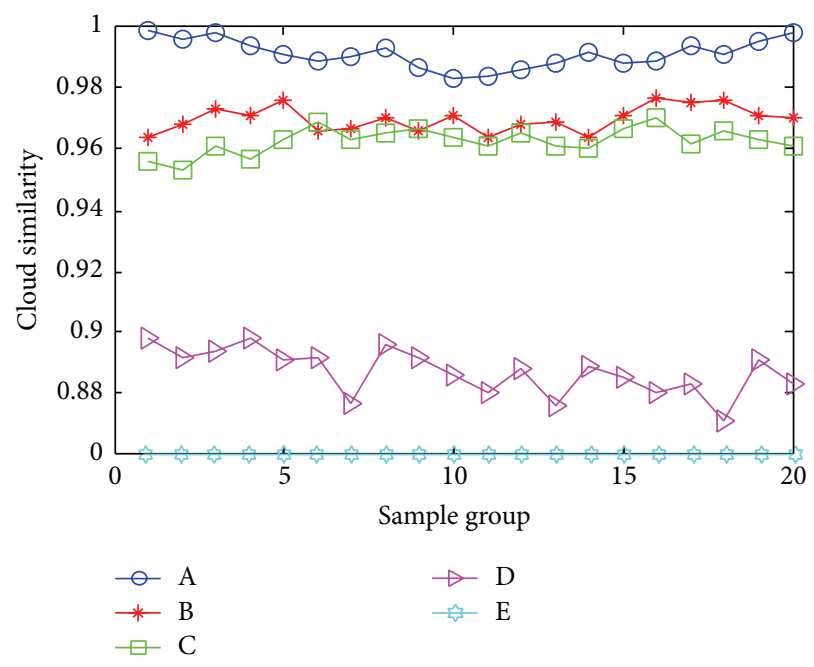

FIGURE 11: The fitting curves of cloud similarity values of 20 groups of normal samples.

TABLE 3: Comparison of fault diagnosis results.

\begin{tabular}{lcc}
\hline \multirow{2}{*}{$\begin{array}{l}\text { Number of testing } \\
\text { samples }\end{array}$} & \multicolumn{2}{c}{ Accuracy rate \% } \\
& $\begin{array}{c}\text { EEMD-CSM-MME- } \\
\text { SVM }\end{array}$ & $\begin{array}{c}\text { EEMD-MI-MME- } \\
\text { SVM }\end{array}$ \\
\hline 120 & 99.10 & 78.33 \\
180 & 99.16 & 78.40 \\
240 & 99.24 & 78.54 \\
300 & 99.33 & 78.61 \\
360 & 99.33 & 78.61 \\
\hline
\end{tabular}

concluded that this EEMD-CSM-MME method of feature extraction is better, and it can improve the accuracy of fault diagnosis of rolling bearings. 


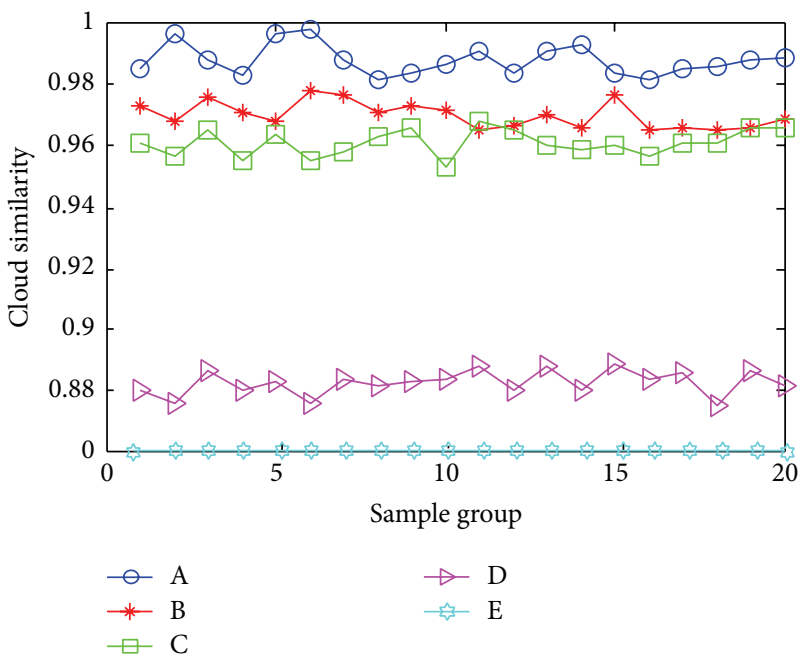

FIGURE 12: The fitting curves of cloud similarity values of 20 groups of fracture samples.

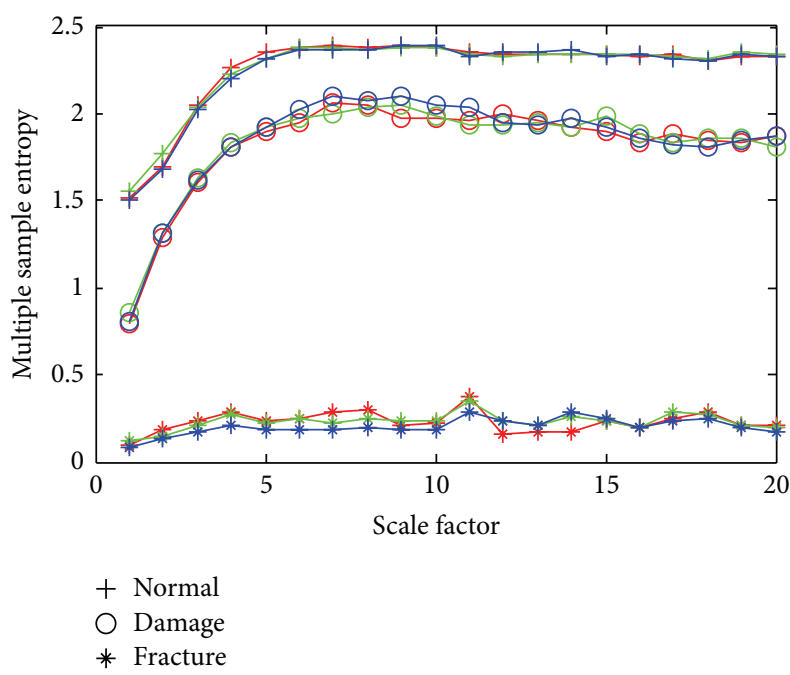

FIGURE 13: The change curves of MME under 3 states.

\section{Conclusion}

In this paper, the Cloud Similarity Measurement is used to select the sensitive IMF; this method has been proved with high accuracy and could overcome the misjudgment, after the simulation experiments. It is more accurate and it can help to overcome the misjudgment caused by mutual information method. It utilizes the experiment platform to collect the normal, damage, and facture failure of AE signal, and it selects the sensitive IMF according to Cloud Similarity Measurement. Then to obtain the fault eigenvalue calculated by Multivariate Multiscale Entropy, it is classified by SVM. The results of the experiments show that the selected sensitive IMFs by CSM are effective; it can also improve the accuracy of feature extraction and fault diagnosis.

This study can be considered as the first investigative step since it concerns a single application of the method to specific

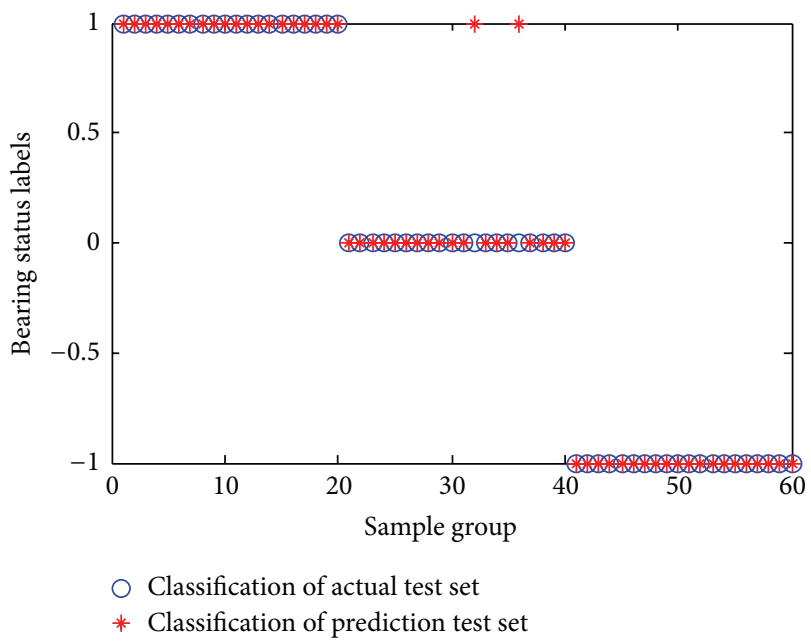

(a) EEMD-CSM-MME-SVM fault diagnosis

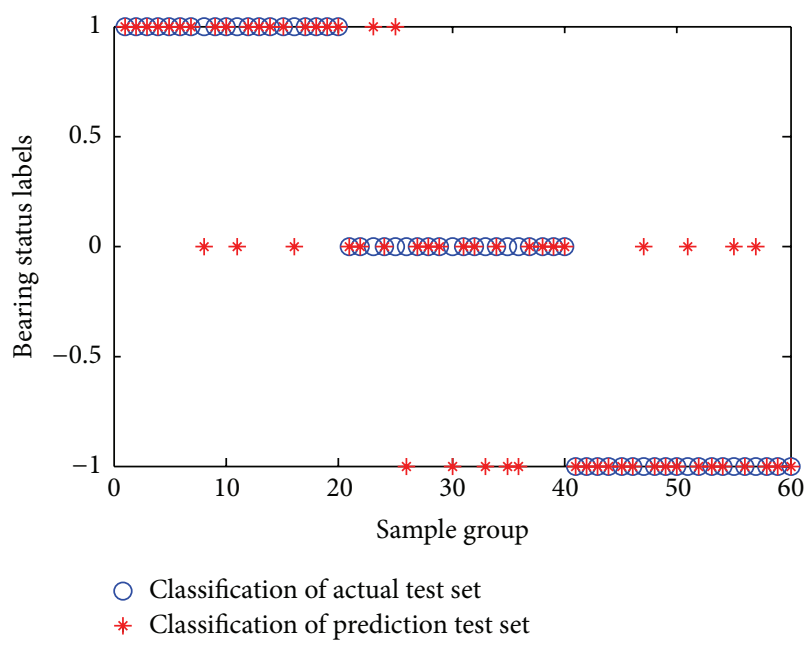

(b) EEMD-MI-MME-SVM fault diagnosis

Figure 14: Two methods on the effect on fault diagnosis.

TABLE 4: Notation list of the mathematical symbols used in the analysis.

\begin{tabular}{lcc}
\hline Mathematical symbol & Definition & Unit \\
\hline$E_{x}$ & Expected value of cloud mode & - \\
$E_{n}$ & Entropy of cloud mode & - \\
$H_{e}$ & Hyperentropy of cloud mode & - \\
$\delta$ & Similarity threshold & - \\
$M$ & Embedding vector & - \\
$\lambda$ & Time lag vector & - \\
$r$ & Tolerance level & - \\
$N$ & Length of time series & - \\
$C$ & Penalty factor & - \\
$\sigma$ & Kernel parameter & - \\
\hline
\end{tabular}

type of bearings and to unique specimens and therefore its effectiveness has to be proved with further investigations. 


\section{Appendix}

See Table 4.

\section{Conflict of Interests}

The authors declare that there is no conflict of interests regarding the publication of this paper.

\section{Acknowledgments}

The research was supported partially by National Natural Science Foundation of China (Grant nos. 51107015 and 60904050) and the science and technology research projects of the Education Department of Heilongjiang Province (12543057).

\section{References}

[1] B.-Y. Li, J.-L. Feng, G. Dai, and W. Li, "Progress in the study of acoustic emission for evaluation of pitting corrosion in metal," Technical Acoustics, vol. 24, no. 3, pp. 170-172, 2005.

[2] M. Elforjani and D. Mba, "Monitoring the onset and propagation of natural degradation process in a slow speed rolling element bearing with acoustic emission," Journal of Vibration \& Acoustics, vol. 130, no. 4, Article ID 041013, pp. 1257-1261, 2008.

[3] M. Elforjani and D. Mba, "Accelerated natural fault diagnosis in slow speed bearings with Acoustic Emission," Engineering Fracture Mechanics, vol. 77, no. 1, pp. 112-127, 2010.

[4] M. Elforjani and D. Mba, "Condition monitoring of slow-speed shafts and bearings with acoustic emission," Strain, vol. 47, no. 2, pp. 350-363, 2011.

[5] A. M. Al-Ghamdi, P. Cole, R. Such, and D. Mba, "Estimation of bearing defect size with acoustic emission," Insight: NonDestructive Testing and Condition Monitoring, vol. 46, no. 12, pp. 758-761, 2004.

[6] A. Choudhury and N. Tandon, "Application of acoustic emission technique for the detection of defects in rolling element bearings," Tribology International, vol. 33, no. 1, pp. 39-45, 2000.

[7] P. Beck, T. P. Bradshaw, R. J. Lark, and K. M. Holford, "A quantitative study of the relationship between concrete crack parameters and acoustic emission energy released during failure," Key Engineering Materials, vol. 245-246, pp. 461-466, 2003.

[8] B. Kilundu, X. Chiementin, J. Duez, and D. Mba, "Cyclostationarity of Acoustic Emissions (AE) for monitoring bearing defects," Mechanical Systems and Signal Processing, vol. 25, no. 6, pp. 2061-2072, 2011.

[9] M. Zhao, L. Li, and B. Cheng, "Wavelet envelope spectrum analysis on acoustic emission signals of rolling bearing fault," Bearing, no. 4, pp. 38-41, 2008.

[10] C. Lilin, L. Aiqun, and D. Yang, "Combined application of acoustic emission and wavelet packet analysis on damage condition monitoring of structures," Journal of Vibration, Measurement \& Diagnosis, vol. 32, no. 4, pp. 591-595, 2012.

[11] F. Xu and Y. Liu, "Feature extraction and classification method of acoustic emission signals generated from plywood damage based on EMD-SVD," Journal of Basic Science and Engineering, vol. 22 , no. 6, pp. 1238-1247, 2014.

[12] Z. Wu and N. E. Huang, "Ensemble empirical mode decomposition: a noise-assisted data analysis method," Advances in Adaptive Data Analysis, vol. 1, no. 1, pp. 1-41, 2009.
[13] Y. Lei, "Machinery fault diagnosis based on improved HilbertHuang transform," Journal of Mechanical Engineering, vol. 47, no. 5, pp. 71-77, 2011.

[14] Z. Zhou, Y.-S. Zhu, Y.-Y. Zhang, C.-F. Zhu, and P. Wang, "Adaptive fault diagnosis of rolling bearings based on EEMD and demodulated resonance," Journal of Vibration and Shock, vol. 32, no. 2, pp. 76-80, 2013.

[15] Y. Shen, L. Wang, and Z. Zhao, "Fault diagnosis for rolling bearing of wind turbine based on improved HHT," Measurement and Control Technology, vol. 32, no. 9, pp. 40-44, 2013.

[16] L. Deyi and D. Yi, Artificial Intelligence with Uncertainty, National Defense Industry Press, Beijing, China, 2005.

[17] L. J. Dai and Z. F. He, "A time series similarity measurement based on cloud model," in Proceedings of the 8th Symposium China Annals of Uncertain Systems, pp. 118-124, 2010.

[18] J. Zhang, R. Yan, R. X. Gao, and Z. Feng, "Performance enhancement of ensemble empirical mode decomposition," Mechanical Systems and Signal Processing, vol. 24, no. 7, pp. 2104-2123, 2010.

[19] X. Dezhi and L. Xiaohui, "Recommendation algorithm of item ratings prediction based on cloud model," Computer Engineering, vol. 36, no. 17, pp. 48-50, 2010.

[20] D. Mitraković, I. Grabec, and S. Sedmak, "Simulation of AE signals and signal analysis systems," Ultrasonics, vol. 23, no. 5, pp. 227-232, 1985.

[21] A.-P. Albert and A.-O. Nii, "A criterion for selecting relevant intrinsic mode functions in empirical mode decomposition," Advances in Adaptive Data Analysis, vol. 2, no. 1, pp. 1-24, 2010.

[22] M. U. Ahmed and D. P. Mandic, "Multivariate multiscale entropy analysis," IEEE Signal Processing Letters, vol. 19, no. 2, pp. 91-94, 2012. 

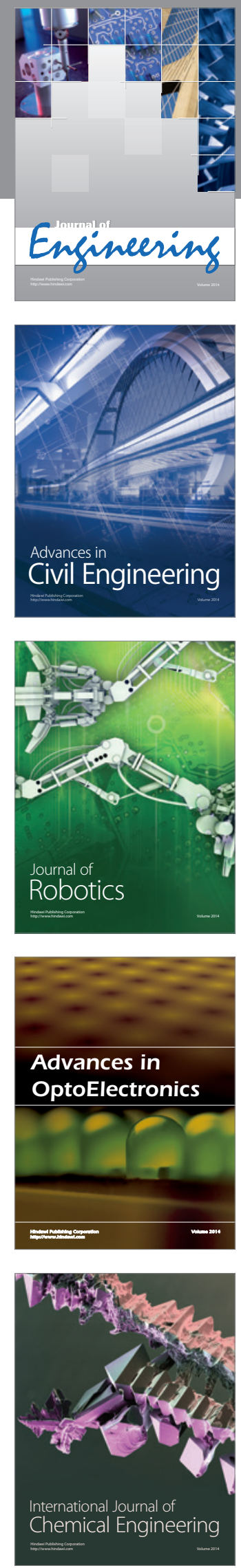

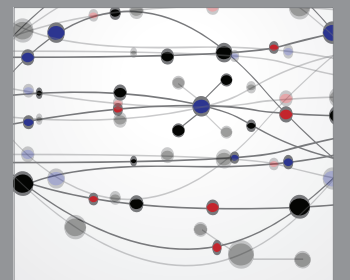

The Scientific World Journal
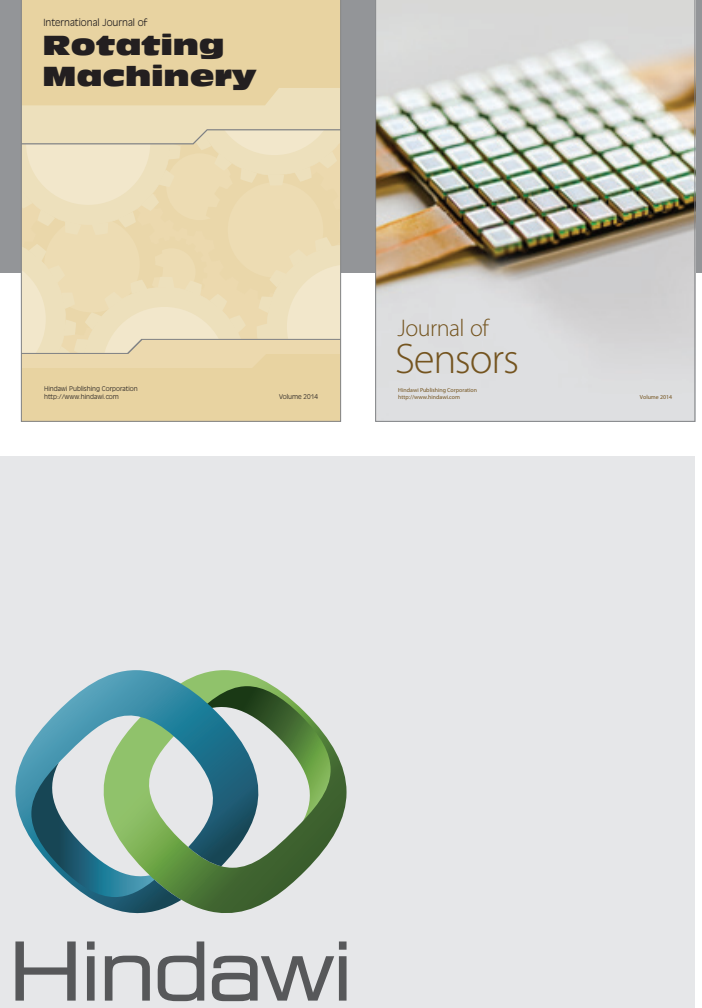

Submit your manuscripts at http://www.hindawi.com
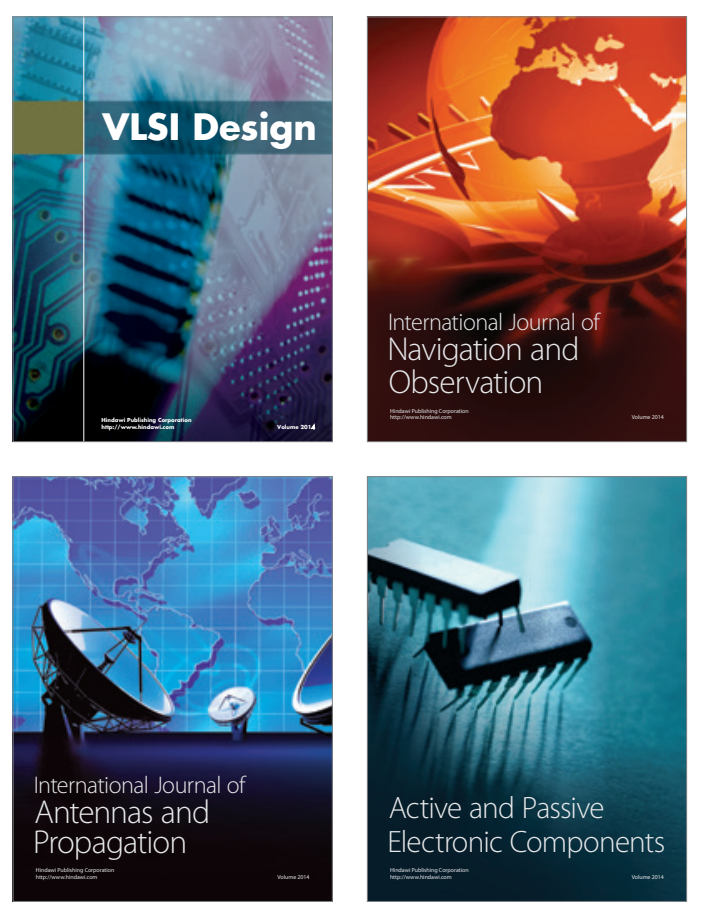
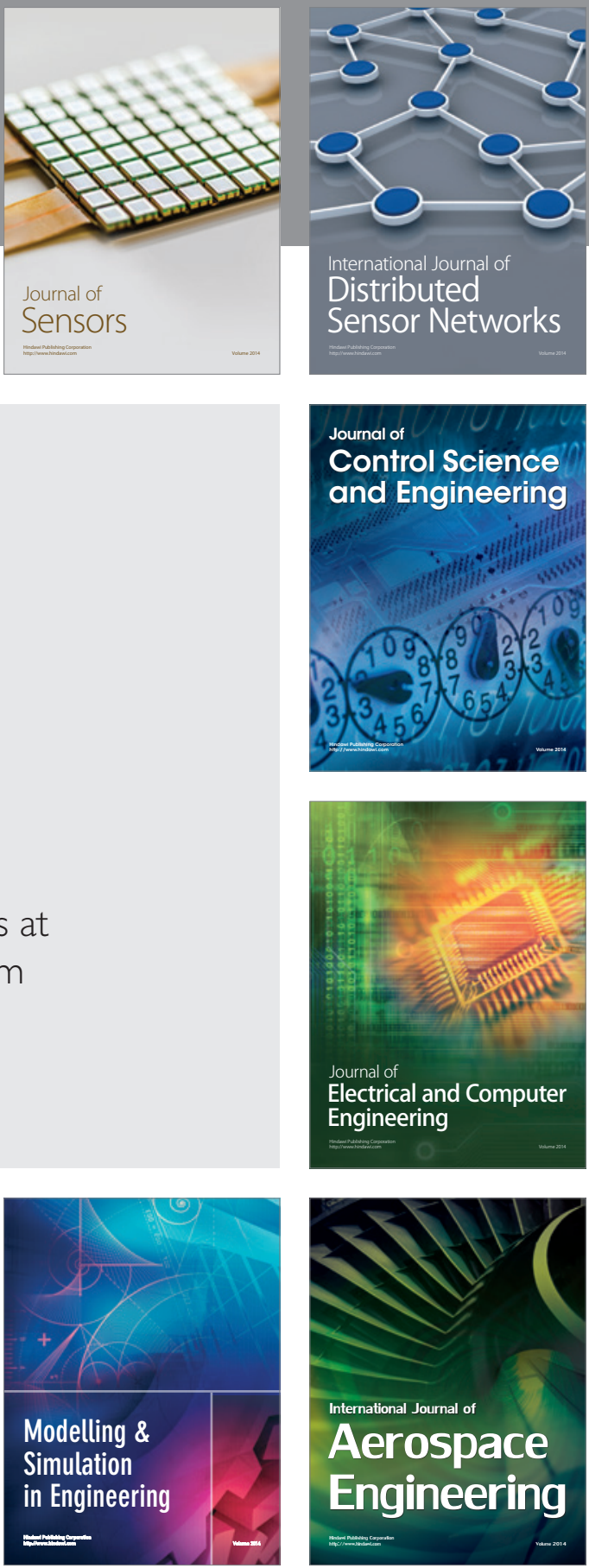

Journal of

Control Science

and Engineering
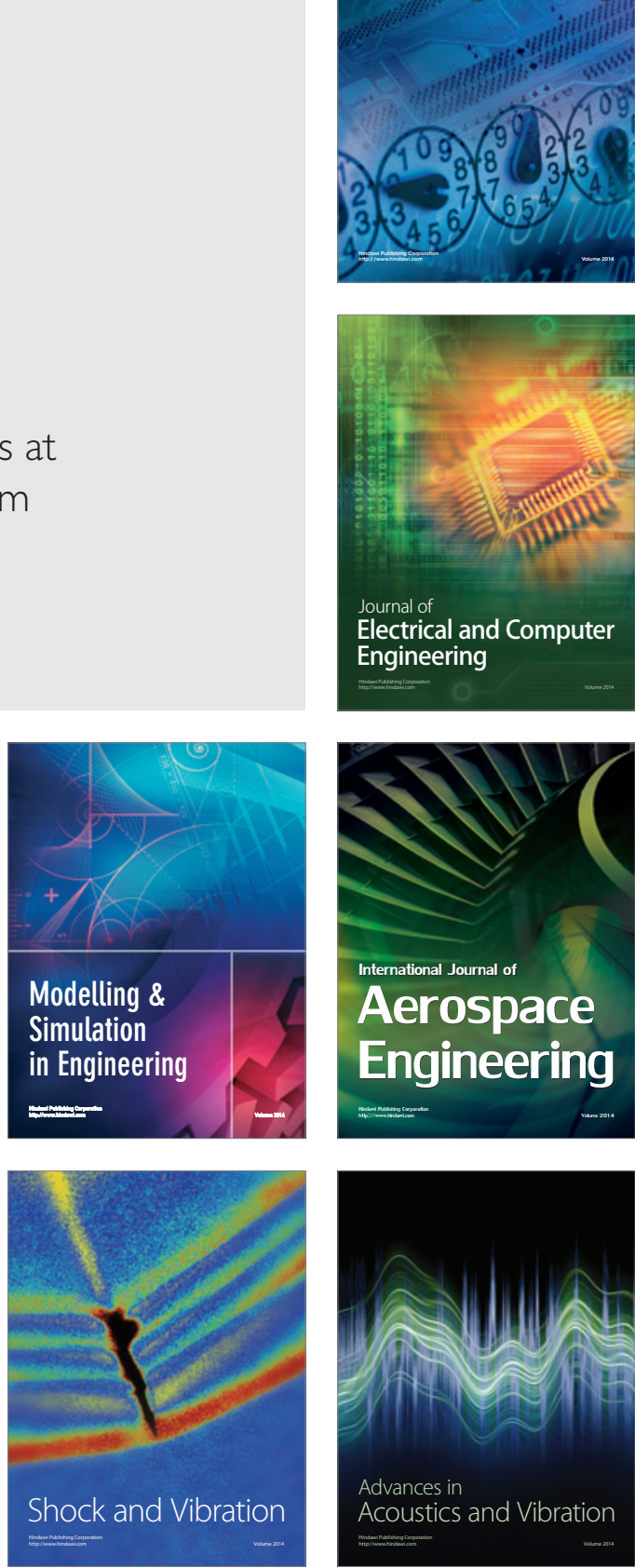